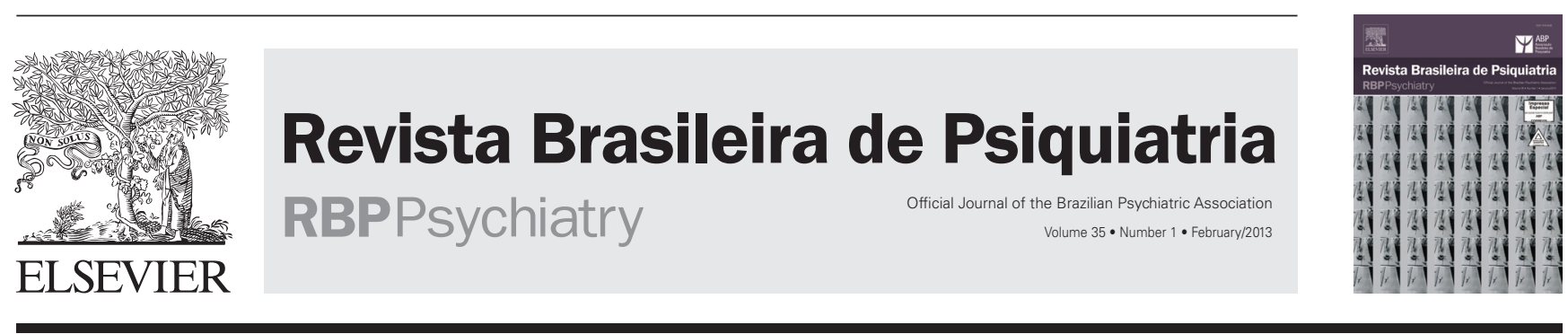

Letter to the Editors

\title{
rTMS to treat social anxiety disorder: a case report
}

Dear Editor,

Social anxiety disorder (SAD) is one of the most common and debilitating types of anxiety disorders. Nevertheless, little attention has been dedicated to the study of the neurobiology underlying SAD until the last decade. ${ }^{1}$ Approximately $25 \%$ of anxiety disorder patients, including SAD patients, are nonresponders to traditional methods of treatment. ${ }^{2}$ Thus, advances in our understanding of the neural basis involved in SAD could lead to new therapeutic options. ${ }^{1}$ One such novel therapeutic option is repetitive transcranial magnetic stimulation (rTMS), a non-invasive procedure whereby a pulsed magnetic field stimulates electrical activity in the brain and depolarizes neurons. ${ }^{2}$ We report a case of a 38 year old single male. The patient signed a consent form and was aware of the experimental protocol (approved by the Ethics Committee at Universidade Federal do Rio de Janeiro) before participation commenced.

The patient was diagnosed with specific SAD (writing in public), without comorbidities according to DSM-IV-TR. The patient was treatment-resistant to a serotonin-specific reuptake inhibitor (SSRI) and cognitive behavior therapy (CBT). A single session of rTMS was administered at $1 \mathrm{~Hz}$ (inhibitory frequency) at 120\% MT for 25 min (1,500 pulses) over the right ventromedial prefrontal cortex (VmPFC). The vmPFC represents the most promising target for rTMS because this structure is consistently activated in SAD. ${ }^{1}$ The right vmPFC is located near the Fp2 position according to an EEG-international 10/20 electrode scalp positioning system. Through this system, satisfactory activation of cortical areas may be reached reliably on a larger scale. ${ }^{3}$

Assessments were carried out pre- and post-treatment using the Beck Depression Inventory (BDI), Beck Anxiety Inventory (BAI) and Social Skills Inventory (SSI). Pre-treatment results were the following: BDI 18 (moderate), BAI 21 (moderate). Post-treatment results were the following: BDI 19 (moderate), BAI 5 (minimal). At a 2-month follow-up, we obtained the following results: BDI 13 (minimal) and BAI 13 (mild) (Figure $1 \mathrm{~A}$ ). With respect to SSI, there were changes in social skill performance in 12 ( 8 positives and 4 negatives) of 32 issues between the pre-treatment and the post-treatment periods and in 16 (10 positives and 6 negatives) of 32 issues between the post-treatment and the follow-up periods (Figure 1B).
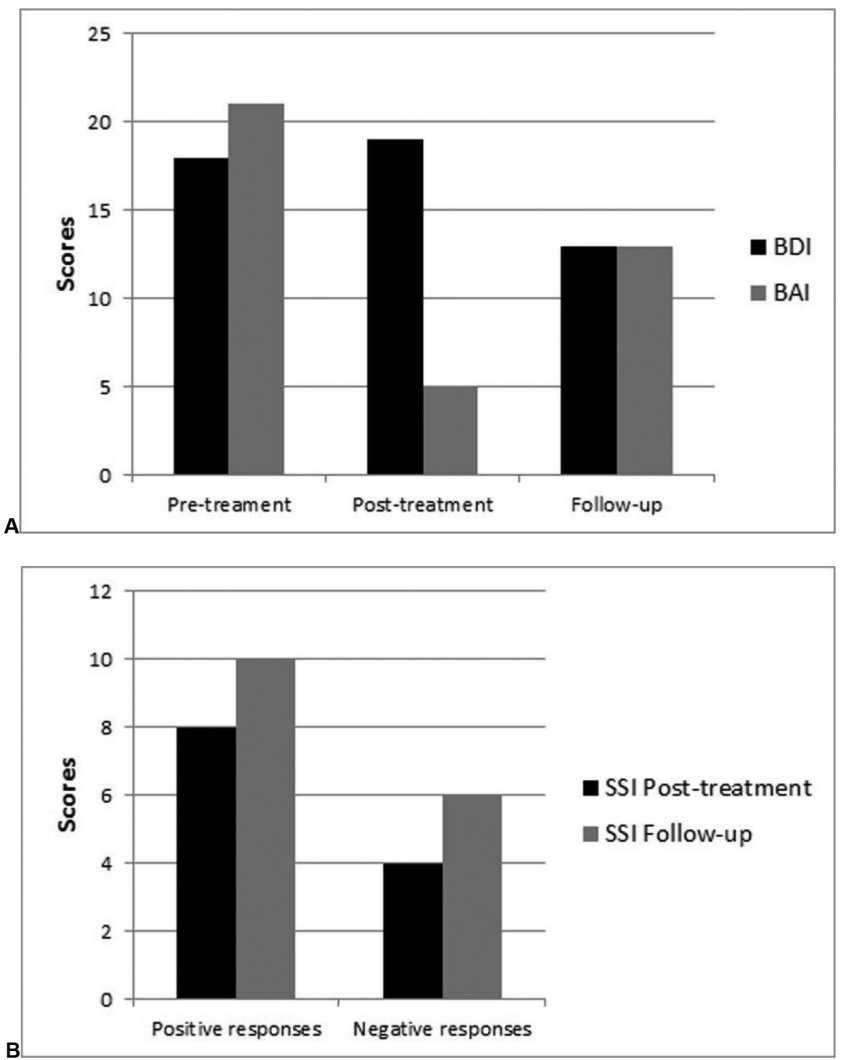

Figure 1 Scores of Beck Depression Inventory (BDI), Beck Anxiety Inventory (BAI) and Social Skills Inventory (SSI) related to rTMS application. (A) Scores of BDI and BAI at pre- and post-treatment and at follow-up. (B) Scores of positive and negative responses to SSI at post-treatment and follow-up. 


\section{Discussion}

Prior to the rTMS session, the patient presented a moderate level of anxiety, reporting for example, an inability to relax, nervousness, and a fear of the worst happening (areas most highly rated on $\mathrm{BAI}$ ). One week after the rTMS session, the patient showed large reduction in anxiety symptoms compared to pre-treatment. With regard to social skills performance, the patient showed a mild reduction post-treatment compared to pre-treatment. The patient reported a greater likelihood to join conversations, to have a sense of humor, and to offer help to peers (areas most highly rated on SSI). These findings suggest that $1 \mathrm{~Hz}$ rTMS over the vmPFC (a brain area responsible for emotional regulation $)^{4}$ promoted the reinterpretation and reprocessing of events regarding the patient's anxiety level and social skills performance in a more controlled and therapeutic manner. During the two-month follow-up, the patient still presented low-level anxiety and similar social skills performance compared to pretreatment with a slight increase compared to post-treatment. However, without a placebo control, these assumptions are merely speculative at this point.

\section{Flávia Paes, ${ }^{1}$ Sergio Machado, ${ }^{1,2,3,4}$ Oscar Arias-Carrión, ${ }^{5}$ Adriana Cardoso Silva, ${ }^{1}$ Antonio Egidio Nardi ${ }^{1}$}

${ }^{1}$ Laboratory of Panic and Respiration, Institute of Psychiatry, Universidade Federal do Rio de Janeiro; National Institute for Translational Medicine,

Rio de Janeiro, Brazil.

2Physical Activity Neuroscience Laboratory,

Physical Activity Sciences Postgraduate Program of Universidade Salgado de Oliveira, Niterói, Brazil. ${ }^{3}$ Institute of Phylosophy, Universidade Federal de Uberlândia, MG, Brazil. ${ }^{4}$ Quiropraxia Program of Faculty of Medical Sciences, Universidad Central, Santiago, Chile. ${ }^{5}$ Movement Disorders and Transcranial Magnetic Stimulation Unit, Hospital General Dr. Manuel Gea Gonzalez, Secretaría de Salud México DF, México.

\section{Disclosures}

Flávia Paes

Employment: Laboratory of Panic and Respiration, Institute of Psychiatry, Universidade Federal do Rio de Janeiro (IPUB/UFRJ), Brazil; National Institute of Translational Medicine (INCT-TM), Brazil.

Sergio Machado

Employment: Physical Activity Neuroscience Laboratory, Physical Activity Sciences Postgraduate Program of Salgado de Oliveira University (UNIVERSO), Niterói, Brazil.

Oscar Arias-Carrión

Employment: Institute of Phylosophy (IFILO), Universidade Federal de Uberlândia (UFU), Minas Gerais, Brazil.

Adriana Cardoso Silva

Employment: Laboratory of Panic and Respiration, Institute of Psychiatry, Universidade Federal do Rio de Janeiro (IPUB/UFRJ), Brazil; National Institute of Translational Medicine (INCT-TM), Brazil.

Antonio Egidio Nardi

Employment: Laboratory of Panic and Respiration, Institute of Psychiatry, Universidade Federal do Rio de Janeiro (IPUB/UFRJ), Brazil; National Institute of Translational Medicine (INCT-TM), Brazil.

* Modest

** Significant

*** Significant. Amounts given to the author's institution or to a colleague for research in which the author has participation, not directly to the author.

\section{References}

1. Freitas-Ferrari MC, Hallak JE, Trzesniak C, Filho AS, Machadode-Sousa JP, Chagas MH, et al. Neuroimaging in social anxiety disorder: a systematic review of the literature. Prog Neuropsychopharmacol Biol Psychiatry. 2010;34(4):565-80.

2. Machado S, Paes F, Velasques B, Teixeira S, Piedade R, Ribeiro $P$, et al. Is rTMS an effective therapeutic strategy that can be used to treat anxiety disorders? Neuropharmacology 2012;62(1):125-34.

3. Herwig $U$, Satrapi $P$, Schönfeldt-Lecuona C. Using the international 10-20 EEG system for positioning of transcranial magnetic stimulation. Brain Topogr. 2003;16(2):95-9.

4. Quirk GJ, Russo GK, Barron JL, Lebron K. The role of ventromedial prefrontal cortex in the recovery of extinguished fear. J Neurosci. 2000;20(16):6225-31. 